\title{
Exploring New Science Domains with Atom Probe Tomography Enabled by an Environmental Transfer Hub
}

Daniel E. Perea ${ }^{1}$, Daniel K. Schreiber ${ }^{2}$, Arun Devaraj ${ }^{3}$, Dallas D. Reilly ${ }^{4}$, Sten V. Lambeets ${ }^{1}$, Elizabeth J. Kautz ${ }^{4}$, Timothy G. Lach², Mark G. Wirth ${ }^{1}$, James E. Evans ${ }^{1}$

1. Earth and Biological Sciences Directorate, Pacific Northwest National Laboratory, Richland, WA USA

2. Energy and Environment Directorate, Pacific Northwest National Laboratory, Richland, WA USA

3. Physical and Computational Sciences Directorate, Pacific Northwest National Laboratory, Richland, WA USA

4. National Security Directorate; Pacific Northwest National Laboratory, Richland, WA USA

* Corresponding author: daniel.perea@pnnl.gov

The liquid/solid and gas/solid interface represents a rich scientific and technological frontier for discovery and innovation in many areas of science. However, the direct nano- to atomic-scale characterization of these interfaces is technically challenging within broad range of analytical tools that require high to ultrahigh vacuum conditions. This challenge has been largely addressed within the electron microscopy (EM) community through the development of unique specimen holders and differentially-pumped vacuum systems that allow researchers to probe, in situ, liquid/solid and gas/solid interfaces using electron probes [1]. However, the direct application of APT to liquid/solid and gas/solid interfaces is not possible and requires the development of unique specimen preparation and handling of cryogenically-frozen specimens and/or work with specimens under environmentally-protected conditions. At PNNL, we have developed unique suite of hardware and experimental protocols for the preparation and handling of environmentallysensitive materials centered around the combination of a unique environmental transfer hub, specimen suitcase device, and modified cryo FIB/SEM capabilities (Fig. 1a) [2-3]. The ability to prepare, manipulate, and transfer specimens under either cryogenic or vacuum conditions thus provides a means to uniquely apply APT analysis to a wide variety of material systems that would not be possible otherwise. Here we describe some specific examples, shown in Figure 1b, of APT analysis applied to temperature sensitive material systems requiring cryo specimen preparation and transfer, as well as air sensitive materials systems requiring vacuum or inert vacuum transfer.

\section{References:}

[1] M Taheri, EA Stach, I Arslan, PA Crozier, BC Kabius, T LaGrange, AM Minor, S Takeda, M Tanase, JB Wagner, R Sharma, Ultramicroscopy 170 (2016), p. 86.

[2] D.E. Perea, SSA Gerstl, J Chin, B Hirschi, JE Evans, Advanced Structural and Chemical Imaging 3 (2017), p. 12.

[3] DK Schreiber, DE Perea, JV Ryan, JE Evans, JD Vienna, Ultramicroscopy 194 (2018), p. 89. [4] The research was performed using EMSL (grid.436923.9), a DOE Office of Science User Facility sponsored by the Office of Biological and Environmental Research. 
(a)

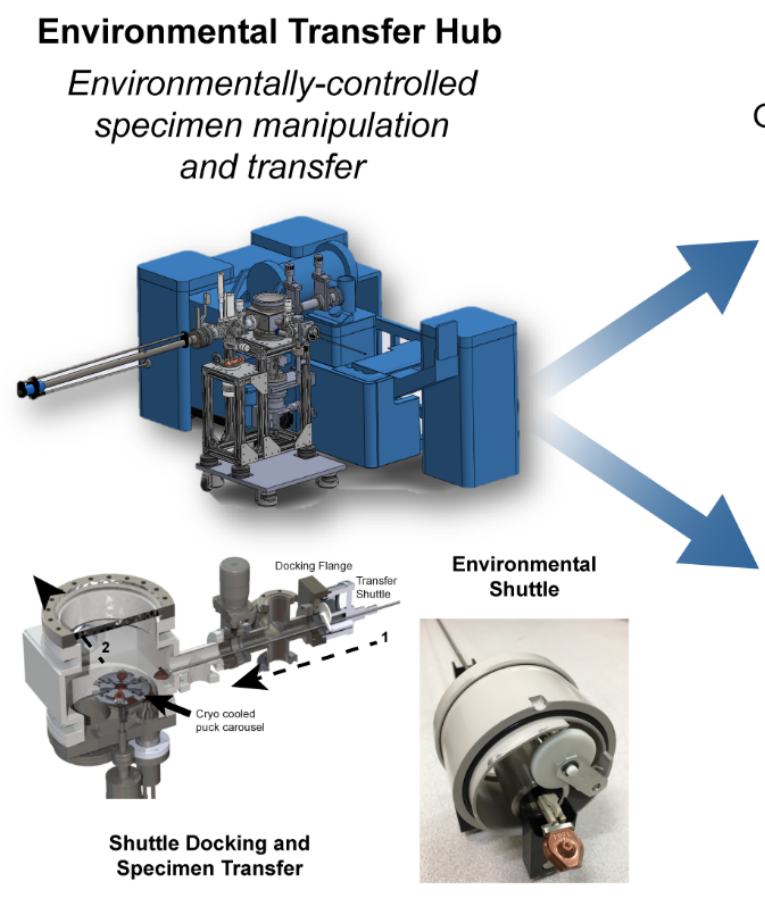

(b) Temperature Sensitive Materials (cryogenic specimen preparation and transfer)

Cells and Biomolecules

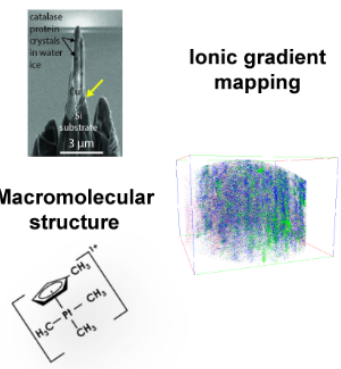

Wasteform Glass

water-filled nanoscale pore structure and composition

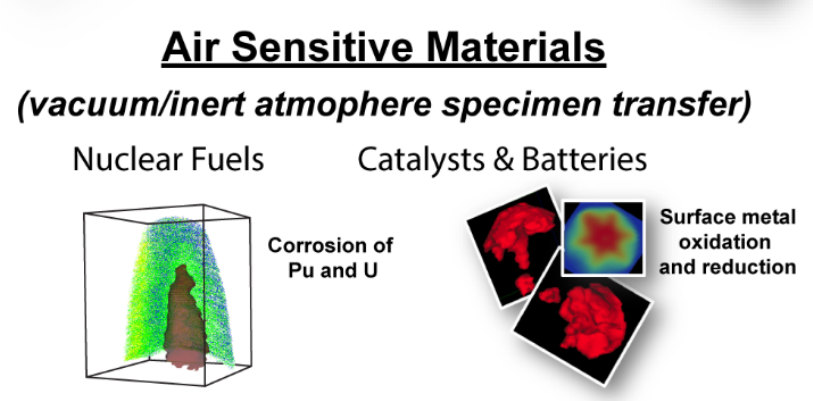

Figure 1. (a) Model representation of the unique Environmental Transfer Hub (ETH) chamber system attached that is attached to the Local Electrode Atom Probe at PNNL. The ETH enables the docking of a modified specimen shuttle to facilitate the transfer of specimens under passive vacuum and temperature control between a modified cryo-FIB/SEM and the Atom Probe. (b) Application of the ETH is enabling the APT analysis of a wide variety of material systems in the fields of biology and materials science. Additionally, the ETH allows the addition of other ancillary devices such as high temperature gas reactor, combined with UHV specimen transfer, provides a means to perform precise surface studies of air sensitive materials such as nuclear, catalyst, and battery materials. 